\title{
EFFICIENCY IN DOCTORAL TRAINING
}

\author{
John Colombo \\ Professor of Psychology and \\ Associate Dean of the Graduate School at the University of Kansas
}

\section{Introduction}

Like most faculty at research institutions, I am a scientist with an active research program, with current projects funded by NIH, NSF, and industry. I am also an educator engaged particularly in graduate training, and I am currently enjoying a role as advisor/mentor to an excellent collection of five doctoral students and a postdoctoral fellow. However, I am also an administrator in both the research (chair of the Lawrence campus IRB), and the academic (associate dean of The Graduate School) domains. Given this breadth of duties, I am privy to multiple perspectives within the academy about many of the things that the academy does. Most notably with regard to this conference, I am acquainted with a number of views on graduate education, and my comments concern this topic.

Most faculty members (including myself) believe that graduate education -and quite especially doctoral education-is central to the mission of the research university. Among the primary functions of the public research university is the generation of new, non-proprietary knowledge and the sustenance of the public intellectual endeavor. The former charge is readily measured by institutions' output of research and scholarly products. Defining success at the latter is a little more difficult to articulate, but I would propose that

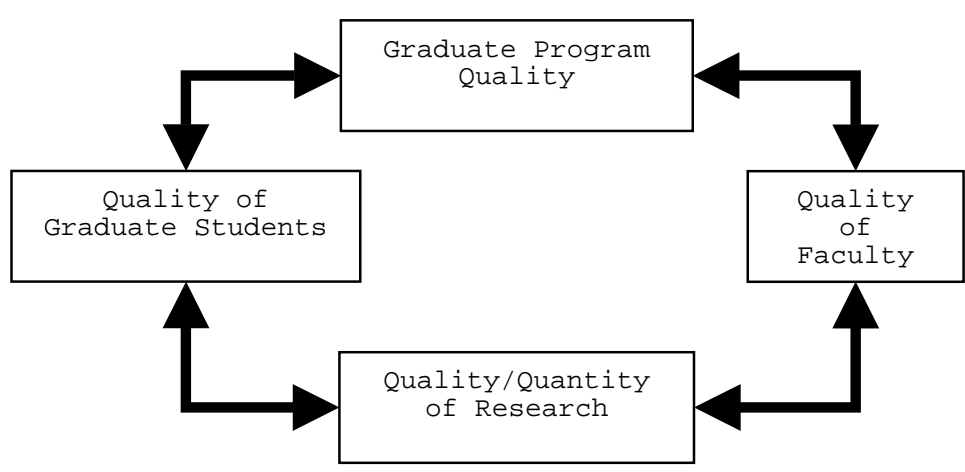

Figure 1. Interplay of research and graduate education this is best operationalized by the training and production of new researchers and scholars. Seeking excellence in these two goals is a highly reciprocal and transactional process; an institution hiring nationally visible and well-funded researchers will (often quickly) gain in its research reputation. This, in turn, will start to attract good graduate students. The presence of good graduate students will make that institution attractive to other desirable faculty candidates, and so on. This cycle is represented in Figure 1. It should be noted that the cycle could run in any causal direction, as indicated by the bi-directional arrows in the figure. 


\section{An Efficiency Analysis of Doctoral Training}

In our discussions of replenishing the professoriate and training the next generation of researchers, there are philosophical and emotional arguments to be made for the pre-eminence of doctoral education as the sine qua non of the research university. However, these are complicated times and with research universities rethinking their priorities, nothing is sacred; all of the functions of the research university must be conceptualized and/or optimized for the greater good of the institution (an academic euphemism for what is more colloquially called "the bottom line"). Given this shift in the paradigm, I wish here to sound a (proactive) alarm for traditional doctoral education. I fear that traditional doctoral education is at risk in these times-yes, even within the modern research university. My judgment of this risk is based largely on the inescapable perception that doctoral training is, in a word, "inefficient." 1 I will suggest some strategies to increase efficiency in doctoral education that do not involve increased resources or funding. Throughout, I will also argue that the adoption of these strategies will contribute to the research mission of the institution and produce graduates who will be highly competitive for the academic and nonacademic marketplaces of the $21^{\text {st }}$ century.

Table 1. Head-to-head comparison of graduate training programs:

A "bottom-line" view

\begin{tabular}{lll} 
Program Variable: & Doctoral & Master's \\
\hline Scope of Student Recruitment & national & local/regional \\
Faculty Prominence & national & unnecessary \\
Program Structure & less & more \\
Institutional Resource Use & more & less \\
Cohort/Program Size & smaller & larger \\
Faculty/Advisor Investment & higher & lower \\
Student Investment & higher & lower \\
Tuition/SCH Generated & less & more \\
\hline "Bottom-Line Efficiency" & low & high
\end{tabular}

Competition in the Age of Efficiency: A Comparison of Doctoral and Master's Programs

Those of us who engage in doctoral training often consider it to be the only kind of graduate education worth doing in higher education. In truth, however, there are really two kinds of graduate education, and the "other" kind is quickly gaining ground in the academic domain-and for good reason. I refer here to the professional or terminal Master's degree program: the professional MBA, MEd, or MSW, or the stand-alone MA degree that prepares students for a clinical profession. Table 1 shows a head-to-head comparison between

\footnotetext{
${ }^{1}$ I use the term "efficiency" here in its classic and generic form; that is, the ratio of resource use (in terms of time, effort, material resource, etc.) to output (doctoral graduates produced).
} 
traditional doctoral education and these master's programs from the bottomline/efficiency point of view. Doctoral education loses quite handily on all counts where one compares resource use to output. The point of this, of course, is not to suggest that we create more of these Master's programs. Rather, I seek to show those of us who engage in doctoral education that, from the administrative point of view, a reasonable case can be made that "business as usual" with regard to this type of training may not be particularly good business after all. In this spirit, I suggest that some proactive steps might be worth considering.

Critics of this analysis will complain that doctoral education is necessarily costly in resources and time because it involves training to extraordinarily high levels of rigor and quality. At best, this is a difficult empirical point to prove, and at worst it belies a reluctance to examine the situation critically. It is worth considering the possibility that the things that make doctoral education inefficient may have little to do with the actual quality of doctoral graduates, and that increasing efficiency will actually improve the research productivity and marketability for our graduates.

\section{Attrition and Time to Degree as Indices of Efficiency}

How does one improve the efficiency of doctoral education? Table 1 above is instructive but, in truth, doctoral training cannot be made to conform to the parameters of master's programs. For example, doctoral training will never represent more than a small blip in credit-hour production and tuition revenue, compared to undergraduate and master's programs. However, I propose that by addressing two related and fundamental problems at the heart of inefficiency in doctoral training - attrition and time to degree-we may see major improvements in overall efficiency and increases in research productivity of both students and faculty.

Attrition. We accept students into graduate programs but the data suggest that many (if not most) drop out of those programs before finishing the terminal degree. This attrition varies somewhat by discipline and institution, but the best nationwide figures I have seen estimate it at about $40 \%$ to $50 \%$. Interestingly, my experience is that many faculty are incredulous when presented with these figures; they estimate attrition to be, at most, $10 \%$. Attrition, of course, represents inefficiency of time and effort on the part of both students and advisors. Students drop out of doctoral programs for various reasons but two reasons in particular stand out in my mind as primary causes. First, students realize that they do not want to engage in the work required for the doctorate. Second, students change their minds and decide that they no longer want to follow the career path for which the doctorate had been necessary or desired.

Time to Degree. Aside from those students who drop out, those students who do complete the doctorate are taking longer to finish their degrees. This also varies by discipline and how one chooses to measure it, but the best 
estimates we have place average time to degree somewhere around 7 to 10 years. Ideally, the doctorate should take four to six years to complete; the extra years needed to complete the doctorate obviously represent inefficiency of time and effort in producing new researchers and scholars. The increase in this variable over the last two decades has been often attributed to lack of graduate funding (e.g., fellowships, assistantships), which may cause the student to take on other forms of employment while earning the degree. There is some evidence to support this, but it is also clear that financial constraints are not the only ones to consider.

\section{Improving Efficiency}

Given these arguments, the obvious steps to take involve (a) attenuating attrition by either reducing it or (more realistically) have students who are going to drop out do it sooner and with a tangible product, and (b) reducing time to degree. In each case, we are looking to facilitate the movement of students through doctoral programs in a timely fashion.

Some think that improved selection/admissions processes would address problems of high attrition and long time to degree. Theoretically, the argument goes that if we were more selective in who gets admitted to doctoral programs, all those admitted would finish the doctorate (and quickly, at that). I believe this idea is wrong on two levels. First, it's wrong philosophically-it would be counterproductive if we are looking to replenish the professoriate or augment the pool of available researchers for the next century. If anything, we should be looking to be more inclusive under these conditions, not less. Second, it's mistaken practically. Can we really predict who will finish a doctorate (quickly) at the point of application from the information provided in the application? As a developmentalist who has tried to predict later outcomes from earlier characteristics, I'm highly skeptical of this.

Instead, I suggest that what we can and should do is improve our chances by improving our administration and advising. Below are some suggestions that seek to do just that. They are grouped by the institutional level at which they might be implemented.

\section{Administrators}

Set-and enforce-reasonable time limits. All research universities have time limits to the completion of the degree. In my experience, these limits can and often are successfully petitioned and extended. Such extension usually comes with the support of the advisor and often with the support of the program. The regular granting of extensions sends an obvious message to students and faculty that the time limits need not be respected, or that they are arbitrary. The enforcement of these limits by administrative units above the department or program would establish a culture change with regard to time to degree. It is 
most effective for such enforcement to come from above the program level, so that advisors avoid the inherent conflicts of interest in serving as both advocate and regulator for their students.

Establish a probationary period for pursuit of the Ph.D. This may be done at the school/college, department, or program level. It can be made clear to students that even though they have been admitted to a Ph.D. program, admission per se is not a guarantee of entitlement toward the degree. Students can be advised that the period leading to, for example, the MA degree (or some equivalent point at the beginning of the doctoral program) is considered to be a "probationary" period. At the end of this period the student's advisor and/or the student's committee will engage in a discussion of the student's progress in the discipline and program, from which a formal recommendation can be made as to whether the student should continue on for the doctorate. This gives programs and students an institutionalized and face-saving "out" for both student and institution (particularly if the period ends with the M.A., where the student will still walk away with a graduate degree) in cases where the student chooses not to continue the work toward the doctorate.

Develop graduate curricula that are both structured and flexible. The doctoral programs with which I have become familiar (either as a faculty member or as an associate dean) are bimodal with respect to coursework requirements. Some are completely unstructured (e.g., 3 required courses), while others have a required coursework load that is particularly burdensome (e.g., 17 required courses). The rationale for the former is that it allows for optimum flexibility. At best, the latter is justified based on the expectation that students will be both broadly and deeply trained ${ }^{2}$; at worst, it serves to populate graduate seminars adequately in the face of university class-size minima.

In programs with little structure, students take too long because they flounder about in their studies (not to mention that faculty in the program cannot expect a reasonable amount of core knowledge in the students enrolled in their graduate seminars). In programs with too much structure, the coursework gets in the way of the development and conduct of the research program that will make the student competitive in the academic marketplace, and often extends time to degree.

It has long been my opinion that required curricula in doctoral programs should be designed for completion by the end of the second year. To me, this translates to no more than 8 required courses, with just electives (chosen by the student as s/he sees fit) remaining beyond this point. This allows enough structure to support students' acclimation to the academic environment at the start of their graduate careers, and then enough time to develop a thoughtful and meaningful research program (and presumably, thoughtful and meaningful

\footnotetext{
${ }^{2}$ Perhaps this is why inter/multidisciplinary programs disproportionately fall into the latter category.
} 
research products, including grant proposals) toward the end of their graduate careers.

Balance doctoral degree requirements between traditional form and current function. A suggestion related to the one described above is a proposal to "scale-down" the traditional, formalized, and somewhat onerous format of degree products. In psychology, for example, the average manuscript length for a typical published empirical report is between 30 and 40 pages, but some theses and dissertations are 200 to 300 pages long. Is the production of a document that is five to ten times the length of the functional output of a research endeavor truly necessary? I wonder whether a student's mastery of the skills of scholarship might be demonstrated in a briefer and more functional format. I am not advocating for 9-page dissertations here, but I am proposing a more moderate length; l'd think that we all would have a sense of what that would be within our own fields. In my own discipline of psychology, this might need be only 50-60 pages.

\section{Advisors}

Make background, expectations, and requirements explicit. In the mid-1990s, I took a month during the summer and developed a handbook for my incoming and continuing graduate students that lays out the rationale for our research program at its most fundamental level, our funding sources, our facilities and sites, my expectations for program requirements and time to degree, as well as general lab policies regarding use/ownership of data, equipment, and facilities. The handbook finishes with a listing of all published research and presentations from the laboratory, showing that student authorship was more a rule than an exception on our products, and that there was a strong expectation for students to generate such products. I take some time to update it each summer; this takes a few hours at most. The handbook, which is given to each student upon their acceptance into my laboratory, establishes a culture of productivity, makes responsibilities and duties clear to all, and defuses potential problems with regard to policy. The point is that the provision of this information works, and I encourage my colleagues to develop something like this as well. Indeed, this kind of step constituted a fundamental recommendation from the 2000 National Studies on Doctoral Education. ${ }^{3}$

Integrate new students into existing lines of research. Many of the advisors I know insist that student projects at both the master's and doctoral level reflect contributions to the discipline that are completely independent of their own. I agree wholeheartedly that this is a goal for the end of the doctoral training sequence. However, I find that the entering doctoral student, however capable and intelligent, cannot readily or promptly conceptualize, design, implement, carry out, and disseminate a meaningful project within the context of a discipline. As such, I work very closely with students on their first research projects (usually

\footnotetext{
${ }^{3}$ See www.grad.washington.edu/envision/project_resources/national_recommend.html.
} 
those used for the completion of the MA degree). In their first years, I will suggest that they take on a programmatic extension of my own of work. If the student so chooses to take responsibility for such an extension (most do, and are usually quite happy to do so), they waste no time floundering for a topic, and they quickly begin working on a piece of research that has a relatively high probability of providing a real research credential. In doing this, the student gains experience conducting, analyzing, and writing up the research; all of this will serve them well as they learn more about the field and then seek a more independent contribution later in their graduate career.

Give graduate students a sense of "belonging"-to the lab, to the institution, and to the field. Research on graduate education has repeatedly shown that greater integration of students into a social structure related to their scholarly work reduces both attrition and time to degree. ${ }^{4}$ There is much that advisors can do to create and/or structure positive social supports within their research units. For example, a hierarchical laboratory structure makes clear each student's role in the laboratory, as well as the means to ascending the hierarchy in the not-too-distant future. Regular social events (even if they are infrequent), whether it is a holiday dinner or an occasional after-hours off-campus lab meeting will also serve to strengthen the social bonds among lab members. In addition, advisors can also do many things to integrate their students into the discipline at large. Major steps in this regard include introducing them to other scholars in the field, and sponsoring/advocating for their work at conference meetings. However, given the sophisticated word processing features available these days, I generate my own laboratory letterhead to use for disciplinary correspondence; listing the names of my graduate student team (in order of seniority) on the letterhead provides a sense (and perhaps some subliminal name recognition) of being part of the field on the national/international level.

Emphasize the timely generation of research products. We may not be particularly good at predicting success in doctoral programs, but we can often discriminate those students who will make good academicians from those who will not. Students who take inordinately long to finish their degrees generally are not good bets to be major contributors to their disciplines. The doctoral students with whom we are concerned here will get and keep an academic position based largely on their ability to generate high-quality research products in a very timely fashion. ${ }^{5}$ Therefore, it makes no sense for advisors to encourage anything but

\footnotetext{
${ }^{4}$ Perhaps this is in part why both indices are so high in the humanities, relative to other fields. It may be that the isolated nature of research in many of the humanities makes it difficult for students to complete or stay with their work. This implies that the creation of improved social networks in these fields would improve time to degree and attrition indices in such disciplines.

${ }^{5}$ I realize that not all doctoral students will seek academic posts, and that not all academic posts will require a high research output for promotion and tenure. The focus of this conference, however, is on replenishing the professoriate and how to train the next generation of researchers. In that spirit, we are speaking here of academic positions with relatively high research expectations. At this time, it is worth noting that such expectations are not uncommon at comprehensive/regional universities.
} 
this particular work ethic in their laboratories. If this is done, it is my experience that the student quite clearly sees a path to the doctorate and beyond, and develops a sense of confidence and expectation of success. Students, of course, often cannot churn out such products (especially during the early parts of their graduate tenure) without the help of their advisors, and so the adoption of this stance within one's laboratory necessarily comes with a cost-the reading, editing, re-reading, and re-editing of (sometimes many) publication drafts and grant proposals. It should be noted, however, that such products have a tremendous up side; in the long run, they will make the laboratory, the program, and the institution more productive in its research mission.

\section{A Personal Record of Graduate Advising}

So, how do these processes work? To help elucidate this point, I refer you to Table 2, which presents my personal record of doctoral advising over the course of 15 years. I present this not as a shining example of graduate success, but rather as a concrete example of some of the issues l've discussed above with regard to doctoral education. The table shows the years that the student $\mathrm{s}$ matriculated in the Ph.D. program (although because I inherited some of these students from other advisors or co-advised them, not all those years represent my tutelage), their fate with regard to the degree, and their last known placement in the workforce.

The first question one has to ask is whether this is a good or adequate record for 15 years of work. If you count, I have seen only 11 students through to some level of completion to this point (although I currently have 5 more on the way). Of the 11 , only 5 completed their doctorates (so the $50 \%$ statistic clearly applies to me). Of the 5, 3 have been placed in true research universities, and 2 in traditional assistant-professor roles. If the goal of doctoral training is that all students should finish, and that all should be placed in nationally prominent or visible positions, then this record is not very good.

At the same time, several things are noteworthy about this list of individuals, owing to policies that I have developed over the years that are concordant with the suggestions made in the sections above. None have left the program after becoming a doctoral candidate (i.e., ABD). The median time to degree for those who finished is 6 years, which is less than the national average for psychology. (Still, some did not finish as quickly as either of us would have liked.) For those who did not complete the doctorate, only one walked away from graduate study without a degree; those who finished M.A.s did so within a minimal period (2-3 years). Finally, those students who left the pursuit of the doctorate generally did so for multiple reasons unrelated to their satisfaction with doctoral training or lack of funding; although these may be failures from the viewpoint of my discipline, they have generally found success elsewhere for which (most of them will tell you) their interrupted doctoral training had prepared them to some degree. 
Table 2. A personal record of doctoral advising

\begin{tabular}{|c|c|c|c|}
\hline & $T T D^{*}$ & Outcome & Placement \\
\hline A & $8 \mathrm{yrs}$ & $\mathrm{PhD}$ & Professor, comprehensive university \\
\hline B & $3 \mathrm{yrs}$ & no degree ${ }^{a}$ & Career in telecommunications for disabled \\
\hline $\mathrm{C}$ & 6 yrs & $\mathrm{PhD}$ & Associate professor, comprehensive university \\
\hline D & 3 yrs & left after $M A^{a, b}$ & Account analyst, telecommunications \\
\hline$E$ & $5 \mathrm{yrs}$ & $\mathrm{PhD}$ & Associate professor, R1 university \\
\hline $\mathrm{F}$ & 2 yrs & left after $M A^{b}$ & Owner, successful daycare center \\
\hline G & $2 \mathrm{yrs}$ & left after $M A^{b}$ & Database manager, social services agency \\
\hline $\mathrm{H}$ & 5 yrs & $\mathrm{PhD}$ & Associate professor and chair, R1 university \\
\hline I & $7 \mathrm{yrs}$ & $\mathrm{PhD}$ & Research associate, R1 university \\
\hline $\mathrm{J}$ & 2 yrs & left after $M A^{b}$ & Personnel manager, telecommunications \\
\hline K & 2 yrs & left after $M A^{b, c}$ & Obtained $\mathrm{PhD}$ in a related program \\
\hline
\end{tabular}

Reasons for leaving: a offered high-paying position in industry, ${ }^{b}$ changed mind about academic/research career, ${ }^{c}$ wanted doctorate in a clinical field

\section{Summary}

Doctoral training at the research university is an inefficient process. Despite its apparent centrality to the functions of the research university, its inefficiency makes it a vulnerable target in the contexts of the current economy and institutional mind-sets that feature bottom-line thinking. It will not be possible to entirely change the inefficient nature of doctoral training but it should be possible, at least to some extent, to meet the demands of the changing university environment halfway. And if, in meeting these demands halfway, it is possible to increase research productivity and reputation (and theoretically the direct and indirect grant funds that are virtual byproducts of such an increase), then the "bottom-line" issue is readily addressed as well. Many faculty will claim that the answer to all problems in graduate training lies with increased funding; in my view, funding has a considerable weight in the doctoral training equation, but it is by no means the sole term in the equation.

The apparently high levels of attrition within doctoral education may not be reducible, but it seems likely that such attrition can be recast by placing some emphasis on reducing the time to attrition (i.e., ensuring that if students drop out, they do so after minimal investment) and on documenting that the placements of students who do drop out can be at least in part attributed to the skills they acquired during their interrupted doctoral training.

It also seems quite likely that time to degree can be reduced in a number of ways that will also enhance the research mission of the institution. What I have outlined above is a combination of structuring the experience of doctoral students in terms of coursework, research, and laboratory social structure, and creating a culture of generating timely research products. If these suggestions 
work, all of the stakeholders in doctoral education (students, advisors, institutions, and disciplines) stand to profit handsomely.

\section{Acknowledgements}

A number of scholars and administrators (too many to name) contributed to the development of thought represented here, but I am especially grateful to Patricia Hawley, Todd Little, and Janet Frick for their comments on an earlier draft of this manuscript. 